\title{
Finite dimensional imbeddings of harmonic spaces
}

\author{
K. Ramachandrantand A. Ranjan
}

June 26, 2021

\begin{abstract}
In a noncompact harmonic manifold $M$ we establish finite dimensionality of the eigenspaces $V_{\lambda}$ generated by radial eigenfunctions of the form $\cosh r+c$. As a consequence, for such harmonic manifolds, we give an isometric imbedding of $M$ into $\left(V_{\lambda}, B\right)$, where $B$ is a nondegenerate symmetric bilinear indefinite form on $V_{\lambda}$ (analogous to the imbedding of the real hyperbolic space $H^{n}$ into $R^{n+1}$ with the indefinite form $\left.Q(x, x)=-x_{0}^{2}+\sum x_{i}^{2}\right)$. Finally we give certain conditions under which $M$ is symmetric.
\end{abstract}

\section{Introduction}

Let $(M, g)$ be a riemannian manifold. $(M, g)$ is said to be harmonic if it's density function $\omega_{p}=\sqrt{\left|\operatorname{det}\left(g_{i j}\right)\right|}$ in a normal coordinate neighbourhood around each point $p$ depends only on the geodesic distance $r(p,$.$) . Let \left(r_{p}, \phi\right)$ be polar coordinates around $p$, where $r_{p}(q)=r(p, q)$ is the geodesic distance between $p$ and $q$ and $\phi$ represents a point on the unit sphere in the tangent space $T_{p}(M)$ at $p$. In this system the density is

$$
\Theta_{p}=r^{n-1} \omega_{p}
$$

\footnotetext{
*supported by National Board for Higher Mathematics, DAE, INDIA
} 
A riemannian manifold is said to be globally harmonic if the density $\Theta_{p}$ is a radial or a spherically symmetric function around each point $p \in M$ i.e it depends only on the vaiable $r$ and can thus be written as $\Theta_{p}(r)$. The function $\Theta_{p}(r)$ does not depend on the point $p$, since $\omega_{p}(q)=\omega_{q}(p)$ i.e the function $\omega_{p}$ itself is independent of the point $p$.

Let $(M, g)$ be a harmonic manifold and let $\Delta$ be the laplacian on $M$. Consider a nonzero eigenvalue $\lambda$ and the corresponding eigen space $V_{\lambda}$. If $M$ is compact, $V_{\lambda}$ is known to be finite dimensional. In this case Besse [1] constructed isometric imbedding of $M$ into $V_{\lambda}$. This immersion was crucially used by Szabo [4] in his proof of the Lichnerowicz conjecture, which asserts that Harmonic manifolds are symmetric. Szabo proved the conjecture for compact harmonic manifolds with finite fundamental group. In his paper he generalised Besse's imbedding theorem to give isometric immersions of any harmonic manifold $M$ into $L^{2}(M, g)$, an infinite dimensional space. In particular he recovers Besse's imbedding for compact harmonic manifold. The eigen spaces $V_{\lambda}$ of noncompact manifolds, in particular noncompact harmonic manifolds need not be finite dimensional. Nevertheless the problem of imbedding $M$ into a finite dimensional vector space albeit with an indefinite metric is important and has not been addressed (analogous to the imbedding of the real hyperbolic space $H^{n}$ into $R^{n+1}$ with the indefinite metric $\left.Q(x, x)=-x_{0}^{2}+\sum x_{i}^{2}\right)$. We prove a partial result in this direction.

Theorem 1 Let $(M, g)$ be non-compact harmonic manifold. Let $0 \neq \lambda$ be an eigenvalue of the Laplacian $\Delta$. Assume that all the averaged eigenfunctions of $\Delta$ corresponding to $\lambda$ are of the form $\cosh r+c$, where $c$ is a constant and $r$ is the distance function from the point of averaging. Let $V_{\lambda}$ be the eigen subspace generated by radial eigen functions. Then

1. $V_{\lambda}$ is finite dimensional and

2. there exists a symmetric nondegenerate bilinear form $B$ on $V_{\lambda}$ and an isometric imbedding of $M$ into $\left(V_{\lambda},-B\right)$.

Moreover in this case the volume density is

$$
\Theta(r)=2^{n-1}\left(\sinh \frac{r}{2}\right)^{n-1}\left(\cosh \frac{r}{2}\right)^{n-1-\frac{4}{3}(k+n-1)} .
$$

Also $c$ and $\lambda$ are given by

$$
c=\frac{k+n-1}{\frac{n}{2}+1+k}
$$


and

$$
\lambda=-\frac{2}{3}\left(\frac{n}{2}+1+k\right)
$$

where $\operatorname{Ricci}(M)=-k, k>0$. Finally $f_{p}=\cosh r+c$ has no zeroes on $M$.

Damek and Ricci [2] have given a family of noncompact harmonic spaces which are not symmetric, thus disproving the Lichnerowicz conjecture for the noncompact case. These spaces are called the NA spaces. Though these spaces are non-symmetric, they have an eigen function of the form $\cosh r+C$. These are the only known examples of nonsymmetric harmonic spaces. Thus our theorem covers all the known cases of harmonic manifolds. Nevertheless we strongly believe that our theorem is true for all harmonic spaces.

\section{The imbedding of $M$ into $\left(V_{\lambda},-B\right)$}

Before proving the theorem we make a simple but useful observation.

Observation : Let $V$ be a vector space, $S \subseteq V$ a set such that $V=\operatorname{span} S$. Let $\tilde{B}: S \times V \rightarrow R$ be a map such that

1. $\tilde{B}$ is linear in the second variable, i.e, $\tilde{B}(s,$.$) is linear on V$ for all $s$ in $S$.

2. $\tilde{B}(s, t)=\tilde{B}(t, s) \forall s, t \in S$.

then there exists a unique bilinear form $B: V \times V \rightarrow R$ which extends $\tilde{B}$.

Proof : Define

$$
B\left(\sum_{i} a_{i} s_{i}, v\right)=\sum_{i} a_{i} \tilde{B}\left(s_{i}, v\right)
$$

\section{Proof of the Theorem :}

Let $x$ be any point of $M$ and let $B_{x}$ be the unit ball around $x$. Let $C^{\infty}\left(B_{x}\right)$ be the space of smooth functions on $B_{x}$ with the sup norm.

Consider the restriction map

$$
\Psi: V_{\lambda} \rightarrow C^{\infty}\left(B_{x}\right) ;\left.f \mapsto f\right|_{B_{x}}
$$

Due to analyticity $\Psi$ is injective. We will show that $\operatorname{Im} \Psi$ is finite dimensional. This will prove that $V_{\lambda}$ is finite dimensional. 
Let $f$ be an eigenfunction for the eigen value $\lambda$ and let $f_{p}$ denote the averaged eigen function around $p$. We denote by $C=V_{\lambda}^{+}$the cone generated by $f_{p}, p \in M$, i.e $V_{\lambda}^{+}=\left\{\sum_{i} a_{p} f_{p}: a_{p} \geq 0, p \in M\right\}$ and only finite sums are taken.

claim : $V_{\lambda} \cap B_{x}$ is finite dimensional, i.e $\operatorname{Im} \Psi$ is finite dimensional.

proof : Let $h=\sum_{1}^{k} a_{p_{i}} f_{p_{i}} \in C$. Since $f_{p_{i}}=\cosh d\left(p_{i},.\right)$

$$
\nabla h=\sum_{1}^{k} a_{p_{i}} \sinh d\left(p_{i}, .\right)
$$

Hence

$$
\begin{aligned}
|\nabla h|^{2} & =\left(\sum a_{p_{i}} \sinh d\left(p_{i}, .\right)\right)^{2} \\
& \leq\left(\sum a_{p_{i}} \cosh d\left(p_{i}, .\right)\right)^{2} \\
& =|h|^{2}
\end{aligned}
$$

We consider the space $X=C^{\infty}\left(B_{x}\right) \cap V_{\lambda}^{+}$. Since $|\nabla h|_{B_{x}}|\leq| \nabla h \mid$, the above inequality shows that for $h \in X,|\nabla h| \leq|h|$ holds. This shows the following : If $W$ is a open ball in $V_{\lambda}^{+}$such that $W \cap C^{\infty}\left(B_{x}\right)$ is a bounded family then it is a pointwise bounded equicontinuous family in $X$. Hence by the Arzela-Ascoli theorem it is relatively compact in $X$.

Now we argue as follows: let $U$ be the open ball around the origin in $V_{\lambda}$. Choose a $g$ in $V_{\lambda}$ such that $U \subset C-g$ i.e $U-g \subset C$. The above argument shows that $U-g \cap C^{\infty}\left(B_{x}\right)$ is relatively compact in $V_{\lambda} \cap C^{\infty}\left(B_{x}\right)$, hence $V_{\lambda} \cap C^{\infty}\left(B_{x}\right)$ is finte dimensional and the claim is proved.

Let $S=\left\{f_{p}: p \in M\right\}$ then $V_{\lambda}=S p a n S$. Define

$$
\begin{gathered}
\tilde{B}: S \times V_{\lambda} \rightarrow R \text { by } \\
\tilde{B}\left(f_{p}, \sum \alpha_{q} f_{q}\right)=\sum \alpha_{q} f_{q}(p)
\end{gathered}
$$

Then by the above observation $\exists ! B: V_{\lambda} \times V_{\lambda} \rightarrow R$, which extends $\tilde{B}$.

claim: $B$ is symmetric and nondegenerate.

proof: Clearly $B$ is symmetric. Nondegeneracy of $B$ can be established as follows. Let $h \in \operatorname{Ker} B$, then

$$
B\left(h, f_{q}\right)=0 \forall q \in M \text { i.e, } h(q)=0 \forall q \in M
$$

Now define the map

$$
\Phi: M \rightarrow\left(V_{\lambda},-B\right) \text { by }
$$




$$
\Phi(p):=\Phi_{p}=\cosh d(p, .)
$$

Note that $\Phi(M)$ is contained in $\{B(h, h)=1\}$ for let $\gamma(t)$ be a geodesic in $M$ then

$$
B\left(\Phi_{\gamma(t)}, \Phi_{\gamma(t)}\right)=\Phi_{\gamma(t)} \gamma(t)=1
$$

Further

$$
B\left(\Phi_{\gamma(t)}, \Phi_{\gamma(t)}\right)=1
$$

gives on twice differentiating,

$$
\begin{gathered}
\left.B\left(\Phi_{\gamma(t)}^{\prime}, \Phi_{\gamma(t)}^{\prime}\right)\right|_{t=0}+\left.B\left(\Phi_{\gamma(t)}^{\prime \prime}, \Phi_{\gamma(t)}\right)\right|_{t=0}=0 \\
\text { i.e }\left.B\left(\Phi_{\gamma(t)}^{\prime}, \Phi_{\gamma(t)}^{\prime}\right)\right|_{t=0}+B\left(\left.\Phi_{\gamma(t)}^{\prime \prime}\right|_{t=0}, \Phi_{\gamma(0)}\right)=0
\end{gathered}
$$

But, since $\Phi_{\gamma(t)}=\cosh d(\gamma(t)), \Phi_{\gamma(t)}^{\prime \prime}=\cosh d(\gamma(t),$.$) and hence$

$$
B\left(\left.\Phi_{\gamma(t)}^{\prime \prime}\right|_{t=0}, \Phi_{\gamma(0)}\right)=1
$$

Therefore

$$
\left.B\left(\Phi_{\gamma(t)}^{\prime}, \Phi_{\gamma(t)}^{\prime}\right)\right|_{t=0}=-1
$$

This shows that $\Phi$ gives an isometric immersion of $M$ into $\left(V_{\lambda},-B\right)$. Finally, since $\cosh r$ is a monotone function, $\Phi_{p} \neq \Phi_{q}$ for $p \neq q$. Thus we get an imbedding of $M$ into $\left(V_{\lambda},-B\right)$. This imbedding is nondegenerate since the image of $M$ is not contained in a lower dimensional subspace of $V_{\lambda}$.

We now compute the densities of these harmonic manifolds. Let $(r, \phi)$ be polar coordinates around any point $p$ of $M$ and $f$ be any smooth function on $M$. The Laplacian $\Delta$ in these coordinates is given by

$$
\Delta f=\Delta_{r} f-f^{\prime \prime}-\frac{\Theta^{\prime}}{\Theta} f^{\prime}
$$

Where $\Delta_{r}$ is the laplacian on the distance sphere $S_{r}$ and the primes denote derivatives with respect to $r$. Therefore the action of $\Delta$ on a radial function $f$ (around $p$ ) is

$$
\Delta f=-f^{\prime \prime}-\frac{\Theta^{\prime}}{\Theta} f^{\prime}
$$

Hence if $f$ is a radial eigen function for the eigen value $\lambda$, then $f$ satisfies

$$
f^{\prime \prime}+\frac{\Theta^{\prime}}{\Theta} f^{\prime}+\lambda f=0
$$


By our assumption $f(r)=\cosh r+c$. Hence we get

$$
\frac{\Theta^{\prime}}{\Theta}(r) \sinh r=-(1+\lambda) \cosh r-c \lambda
$$

which gives

$$
\Theta(r)=2^{-(1+\lambda)}\left(\sinh \left(\frac{r}{2}\right)\right)^{-(1+\lambda+c \lambda)}\left(\cosh \left(\frac{r}{2}\right)\right)^{-(1+\lambda-c \lambda)}
$$

But as $r \rightarrow 0, \Theta(r) \rightarrow\left(\sinh \left(\frac{r}{2}\right)\right)^{n-1}$, hence we get

$$
1+\lambda+c \lambda=1-n, \text { and } \Theta(r)=2^{(n-1)}\left(\sinh \left(\frac{r}{2}\right)\right)^{n-1}\left(\cosh \left(\frac{r}{2}\right)\right)^{(n-1+2 c \lambda)}
$$

We now use the ledgers formula [1] (see pp. 161), which gives

$$
-\frac{1}{3} \text { Ricci }=\left.\omega^{\prime \prime}(r)\right|_{r=0}
$$

This shows that harmonic manifolds are Einstein, i.e they have constant ricci curvature.

Let $\operatorname{Ricci}(M)=-k, k>0$. Now

$$
\Theta(r)=r^{n-1} \omega(r)
$$

gives

$$
\frac{\Theta^{\prime}(r)}{\Theta(r)}=\frac{n-1}{r}+\frac{\omega^{\prime}(r)}{\omega(r)}
$$

Using the expression for $\Theta$ obtained above, we get

$$
\frac{\omega^{\prime}(r)}{\omega(r)}=-(1+\lambda) \operatorname{coth} r-\frac{c \lambda}{\sinh r}-\frac{n-1}{r}
$$

Differentiating twice and after simplification, we get

$$
-\frac{1}{3} R i c c i=\frac{n-1}{3}+\frac{c \lambda}{2}
$$

which gives

$$
c \lambda=-\frac{2}{3}(k+n-1), \text { but } 1+\lambda+c \lambda=1-n
$$

hence

$$
\lambda=-\frac{2}{3}\left(1+k+\frac{n}{2}\right) \text { and } c=\frac{k+n-1}{1+k+\frac{n}{2}}
$$

Finally the above computations show that

$$
\begin{aligned}
f & =\cosh r+c \\
& =\cosh r-1+\frac{3 n / 2}{1+k+\frac{n}{2}} \\
& =2 \sinh ^{2}\left(\frac{r}{2}\right)+\frac{3 n / 2}{1+k+\frac{n}{2}} \\
& \geq \frac{3 n / 2}{1+k+\frac{n}{2}}
\end{aligned}
$$

Thus $f$ has no zeroes on $M$. This completes the proof of the theorem. 


\section{$3 \quad$ Symmetry of $M$}

In ths section we prove the symmetry of $M$ under some assumptions. Two cases arise.

Case 1. $\mathrm{c}=0$

In this case $M$ is symmetric. The sturm liouville equation

$$
f^{\prime \prime}+\frac{\Theta^{\prime}}{\Theta} f+\lambda f=0
$$

shows that

$$
\Theta(r)=\sinh ^{n-1} r
$$

A result from [3] shows that $M$ is isometric to the real hyperbolic space with constant curvature -1 .

Case 2. $\mathrm{c} \neq 0$

Let $V_{\gamma}$ be the subspace of $V_{\lambda}$ generated by $\left\{f_{\gamma(t)}: t \in R\right\}$.

Lemma 3.1 Let $M$ satisfy the hypothesis of the above Theorem. Assume in addition that $B$ restricted to the subspace $V_{\gamma}$ is nondegenerate. Then $M$ is symmetric.

Proof. Fix an imbedding $\Phi: M \rightarrow V_{\lambda} ; \Phi(p)=f_{p}$. Let $\gamma(t)$ be a geodesic on $M$. Then

$$
B\left(f_{\gamma(t)}, f_{\gamma(0)}\right)=\cosh t+c
$$

Differentiating we get

$$
B\left(f_{\gamma(t)}^{\prime \prime \prime}-f_{\gamma(t)}^{\prime}, f_{\gamma(0)}\right)=0
$$

Since $\gamma(0)$ is an arbitrary point of $M, f_{\gamma(t)}^{\prime \prime \prime}-f_{\gamma(t)}^{\prime} \in V_{\gamma}$ and by our assumption $B$ is nondegenerate on $V_{\gamma}$, the geodesic $\gamma$ satisfies

$$
f_{\gamma(t)}^{\prime \prime \prime}-f_{\gamma(t)}^{\prime}=0
$$

Take $p \in M$ and let $N_{p}$ be the orthogonal complement, w.r.t $B$ of the tangent space $T_{p}(M)$ to $M$. Define

$$
\tau_{p}: V_{\lambda} \rightarrow V_{\lambda}
$$

be the reflection w.r.t the subspace $N_{p}$. $\tau_{p}$ is an isometry of $V_{\lambda}$, it fixes $p$ and reverses the geodesics through $p$. Moreover it also leaves $M$ invariant. hence it induces an isometry on $M$ which is obviously the geodesic involution of $M$. Thus $M$ is a symmetric space. 
The next lemma shows that if $V_{\gamma}$ is 3 -dimensional then $B$ is nondegenerate on it.

Lemma 3.2 Let $M$ satisfy the hypothesis of the theorem. Let $\gamma$ be a geodesic such that $V_{\gamma}$ is 3-dimensional, then $B$ is nondegenerate on $V_{\gamma}$.

Proof. Let $s_{i}=-1,0,1$ be such that $\left\{f_{\gamma\left(s_{i}\right)}\right\}_{1}^{3}$ generate $V_{\gamma}$. Let $g \in V_{\gamma}$ satisfy $B(g, f(\gamma(t)))=0 \forall t$. Put

$$
\begin{aligned}
g & =\sum_{1}^{3} a_{i} f_{\gamma\left(s_{i}\right)} \\
& =\sum_{1}^{3} a_{i} \cosh d\left(\gamma\left(s_{i}\right), .\right)+c \sum_{1}^{3} a_{i}
\end{aligned}
$$

Now $B\left(g, f_{\gamma(t)}\right)=0$ gives $g(\gamma(t)=0 \forall t$, i.e

$$
\sum a_{i} c+\sum a_{i} \cosh \left(s_{i}-t\right)=0 \forall t
$$

Dividing by $\cosh t$ and letting $t \rightarrow \infty$ we see that

$$
\sum a_{i}=0
$$

hence

$$
\sum a_{i} \cosh \left(s_{i}-t\right)=0 \forall t
$$

Expanding and equating the coefficients of $\cosh t$ and $\sinh t$ to 0 we get

$$
\sum a_{i} \cosh s_{i}=0=\sum a_{i} \sinh s_{i}
$$

Substituting the vaues of $s_{i}$ and using the equation $\sum a_{i}=0$ we obtain a system of three equations

$$
\begin{array}{r}
a_{0} \cosh 1+a_{1}+a_{2} \cosh 1=0 \\
-a_{0} \sinh 1+0+a_{2} \sinh 1=0 \\
a_{0}+a_{1}+a_{2}=0
\end{array}
$$

But the coefficient matrix of the above system is nonsingular, hence $a_{i}=0 \forall i$, i.e $g \equiv 0$ or $B$ is nondegenerate.

Combining the above two lemmas one sees that $M$ is symmetric if $V_{\gamma}$ is 3-dimensional. 


\section{Summary}

For manifolds which satisfy the hypothesis of the theorem the density $\Theta(r)$ is given by

$$
\Theta(r)=2^{n-1}\left(\sinh \frac{r}{2}\right)^{n-1}\left(\cosh \frac{r}{2}\right)^{n-1-\frac{4}{3}(k+n-1)} .
$$

Put $b=n-1-\frac{4}{3}(k+n-1)$. By the Bishop-Gromov comparison theorem $b$ satisfies $0 \leq b \leq n-1$.

1. If $b=n-1, \Theta(r)=\sinh ^{n-1} r$ and hence $M$ is isometric to the real hyperbolic space. In this case $c=0$.

2. If $b=0, \Theta(r)=2^{n-1}\left(\sinh \frac{r}{2}\right)^{n-1}$. Again $M$ is isometric to the real hyperbolic space. But in this case $c \neq 0$ and the eigen function $f_{p}=\cosh r+c$ becomes the eigen function for the next eigen value.

3. All the other harmonic spaces which satisfy the hypothesis of the theorem, in particular the $N A$ spaces have $0<b<n-1$.

Question Is $b$ an integer?, in that case are all harmonic spaces homogeneous? 


\section{References}

[1] A.L.Besse, Manifolds all of whose geodesics are closed, Springer, Berlin, 1978.

[2] E.Damek and F.Ricci, A class of nonsymmetric harmonic riemmanian spaces, Bulletin AMS, 27(1992), pp. 139-142.

[3] K. Ramachandran and A. Ranjan, Harmonic manifolds with some specific volume densities, preprint.

[4] Z.I.Szabo, The Lichnerowicz conjecture on Harmonic Manifolds, J. Differential Geometry, 31(1990), pp. 1-28.

[5] Z.I.Szabo, Spectral theory for operator families on Riemannian Manifolds, Proceedings of symposia in Pure Math, 54 (1993), part 3, pp 615-665.

Department of Mathematics,

I.I.T Powai, Mumbai 400 076,

India.

email: kram@ganit.math.iitb.ernet.in

aranjan@ganit.math.iitb.ernet.in 